\title{
THE SIS GREAT CIRCLE EPIDEMIC MODEL
}

\author{
PETER NEAL, ${ }^{*}$ University of Manchester
}

\begin{abstract}
We consider a stochastic SIS model for the spread of an epidemic amongst a population of $n$ individuals that are equally spaced upon the circumference of a circle. Whilst infectious, an individual, $i$ say, makes both local and global infectious contacts at the points of homogeneous Poisson point processes. Global contacts are made uniformly at random with members of the entire population, whilst local contacts are made according to a contact distribution centred upon the infective. Individuals at the end of their infectious period return to the susceptible state and can be reinfected. The emphasis of the paper is on asymptotic results as the population size $n \rightarrow \infty$. Therefore, a contact process with global infection is introduced representing the limiting behaviour as $n \rightarrow \infty$ of the circle epidemics. A branching process approximation for the early stages of the epidemic is derived and the endemic equilibrium of a major outbreak is obtained. Furthermore, assuming exponential infectious periods, the probability of a major epidemic outbreak and the proportion of the population infectious in the endemic equilibrium are shown to satisfy the same equation which characterises the epidemic process.
\end{abstract}

Keywords: SIS epidemic; contact process; great circle model; endemic equilibrium; branching process

2000 Mathematics Subject Classification: Primary 92D30

Secondary 60G15; 37G35

\section{Introduction}

Stochastic epidemic modelling has focused in recent years on the analysis of infectious diseases in heterogeneously mixing populations. The prime example is the household model (see [5]), where the population is partitioned into small groups (households) and, whilst infectious, individuals make infectious contacts both with the population at large and at an increased rate with members of their own household. This is a two-level mixing model with global infectious contacts uniformly at random with the whole population and local infectious contacts, uniformly at random, with members of ones household. However, there are other examples of two-level mixing models such as the overlapping groups model [1, Section 6] and the spatially motivated great circle model [4], [5]. The great circle model is the focus of this paper and in contrast to [4] we will study SIS epidemics. That is, we assume that there is a closed population of $n$ individuals equally spaced upon the circumference of a circle with individuals numbered sequentially such that individuals 1 and $n$ are neighbours. The individuals are in one of two states: either susceptible or infectious. A susceptible individual when contacted by an infectious individual becomes infectious for a random period of time (an infectious period) during which it can make infectious contacts. Infectious individuals make global infectious contacts, uniformly at random, with members of the whole population and local infectious

Received 28 November 2007; revision received 15 April 2008.

* Postal address: School of Mathematics, University of Manchester, Alan Turing Building, Oxford Road, Manchester M13 9PL, UK. Email address: p.neal-2@manchester.ac.uk 
contacts according to a contact probability distribution $V^{n}$. That is, the individual contacted by a local infection by individual $i$ is given by $\left(i+V^{n}\right) \bmod n$. At the end of its infectious period, the individual returns to the susceptible state and can be reinfected. A full description is given in Section 2.

Contact processes (see, for example, [6], [8], [9], [10], and [12]) are closely related to the SIS great circle model. The contact process model assumes that there is an infinite population equally spaced in a one-dimensional space, usually taken to be at the points of $\mathbb{Z}$. At any point in time, an individual is either susceptible or infectious. On becoming infected an individual has an exponentially distributed infectious period before returning to the susceptible state. Individual $i$, whilst infectious, makes infectious contacts at the points of a homogeneous Poisson point process with rate $\lambda_{\mathrm{L}}$ and the individual contacted is given by $i+V$, where $V$ is a probability distribution with support in $\mathbb{Z}$. With the notable exception of [12], virtually all attention has been focused upon the nearest neighbour model, where $\mathrm{P}(V=1)=\mathrm{P}(V=-1)=\frac{1}{2}$. The contact process only has local (spatial) infectious spread in contrast to the great circle model. Therefore, in Section 2 a contact process with global infection is introduced for general $V$. This is interesting in its own right, but will prove particularly useful when analysing the limiting behaviour as $n \rightarrow \infty$ of the great circle model.

A key tool in analysing both the contact process with global infection and the great circle model is the susceptibility process which is defined in Section 3 . The susceptibility process is the SIS analogue of the susceptibility set which has been utilised to great affect in analysing SIR twolevel mixing epidemics; see [3] and [4]. Also, in Section 3 the simpler notion of a susceptibility time is introduced. In particular, the susceptibility time is crucial in determining both the probability of a major epidemic and, should an endemic equilibrium exist, the proportion of the population infected in equilibrium.

The paper is structured as follows. In Section 2 a description of both the contact and epidemic processes is given. Then in Section 3 susceptibility processes and susceptibility times are defined. In Section 4 contact processes and, in particular, their endemic equilibrium measure is derived. Moreover, provided that there are infinitely many infectives in the initial population configuration, the population converges to the endemic equilibrium measure as time $t \rightarrow \infty$. In Section 5 the emphasis switches to the SIS great circle epidemic model. A law of large numbers is proved showing that the proportion of the population infectious in the SIS great circle model converges to the proportion infectious in the contact process as the population size $n \rightarrow \infty$. The results of Sections 4 and 5 are applicable if, in the limit as the population size $n \rightarrow \infty$, there are infinitely many initial infectives. Therefore, in Section 6 we derive a branching process approximation for the case where there is only one initial infective. In particular, we find that the susceptibility time plays an important role in determining the probability that a major epidemic outbreak occurs. In Section 7 we specialise the results to the nearest neighbour case and provide a useful self-consistent mean-field approximation for the proportion of the population infectious in the endemic equilibrium. Finally, in Section 8 extensions of the current paper are discussed. In particular, how the concepts of susceptibility processes and times can be used in the analysis of other SIS models.

\section{Outline of the contact and epidemic processes}

A graphical representation of the contact process, $C$, upon $\mathbb{Z}$ following [10] is given. For $i \in \mathbb{Z}$, assign to individual $i$ at site $i$ a time line in $\mathbb{R}$. The time line will be comprised of three Poisson point processes corresponding to global infections $\eta_{i}^{\mathrm{G}}$, local infections $\eta_{i}^{\mathrm{L}}$, and removals $\eta_{i}^{\mathrm{R}}$ having rates $\lambda_{\mathrm{G}} x(t), \lambda_{\mathrm{L}}$, and $\gamma$, respectively. Without loss of generality, we will 
take the removal rate to be $\gamma=1$. Clearly, $\lambda_{\mathrm{G}}$ can be amalgamated into $x(t)$, but it will be convenient for discussions in the epidemic context to have $\lambda_{\mathrm{G}}$ specified separately. Associated with each of the points in $\eta_{i}^{\mathrm{L}}$ are independent and identically distributed random variables distributed according to $V$. For $A=\mathrm{G}, \mathrm{L}, \mathrm{R}$, label the points of $\eta_{i}^{A}$ by $T_{i j}^{A}(j \in \mathbb{Z})$ with

$$
\cdots \leq T_{i,-2}^{A} \leq T_{i,-1}^{A} \leq 0 \leq T_{i, 1}^{A} \leq T_{i, 2}^{A} \leq \cdots .
$$

Let $V_{i j}$ denote the random variable associated with time point $T_{i j}^{\mathrm{L}}$. The contact process is then constructed as follows. Let $\eta_{i}^{\mathrm{G}}$ represent the points in time at which potential infectious contacts are made with individual $i$. If individual $i$ is susceptible at time $T_{i j}^{\mathrm{G}}$ then individual $i$ becomes infectious at time $T_{i j}^{\mathrm{G}}$, otherwise the contact is ignored. Let $\eta_{i}^{\mathrm{R}}$ represent the points in time at which individual $i$ recovers from the disease and becomes susceptible again, if infectious. Note that, if individual $i$ is susceptible at time point $T_{i j}^{\mathrm{R}}$, the removal time is ignored. Finally, $\eta_{i}^{\mathrm{L}}$ represents the points in time at which individual $i$ makes potential local infectious contacts. If individual $i$ is infectious at time point $T_{i j}^{\mathrm{L}}$ then they make an infectious contact with individual $i+V_{i j}$ whom, if susceptible, becomes infected. On the other hand, if individual $i$ is susceptible at time point $T_{i j}^{\mathrm{L}}$, the local infectious contact is ignored. For simplicity of exposition, we assume that $V$ is symmetric. Also, we assume that $\mathrm{E}\left[V^{2}\right]<\infty$, which ensures that the rate of growth of the local spread of the disease is linear in time; see [12, Equation (3.21)]. Finally, for $i \in \mathbb{Z}$ and $t \in \mathbb{R}$, let $\xi_{i}(t)=1$ if individual $i$ is infectious at time $t$ and $\xi_{i}(t)=0$ otherwise. Let $\xi(t)=\left(\ldots, \xi_{-1}(t), \xi_{0}(t), \xi_{1}(t), \ldots\right)$.

Throughout, we term the infectious state of the population at time 0 as the initial configuration. In the above construction the initial configuration is determined by studying the time lines into the (infinite) past. This can be done using susceptibility processes, which are described in Section 3. However, we will also be interested in the behaviour of the epidemic for any specified initial configuration $\chi=\left(\ldots, \chi_{-1}, \chi_{0}, \chi_{1}, \ldots\right)$, where $\chi_{i}=1(i \in \mathbb{Z})$ if individual $i$ is initially infectious and $\chi_{i}=0$ otherwise. Where $\chi$ is specified, $\xi(0)=\chi$, and, for $t \geq 0$, $\xi(t)$ can be constructed as above using $\chi$ and the time lines in $\mathbb{R}^{+}$with the time lines prior to time 0 being redundant. We allow $\chi$ to be a random variable and, therefore, the former case (scenario 1) where the initial configuration is constructed from the past time lines is an important special case of the latter, general case (scenario 2 ).

For $n \geq 1$, the contact process $C_{n}$ is defined upon $\{1,2, \ldots, n\}$ with individuals 1 and $n$ as neighbours to form a circle. Global infections and removals take place as for the contact process $C$. Local infections are the same except that the individual contacted at the $j$ th local infectious contact by individual $i$ is $\left(i+V_{i j}\right)_{\bmod n}$. For the epidemic process, $E_{n}$, simply take $C_{n}$ and replace $x(\cdot)$ by $Y_{n}(\cdot)$, where $Y_{n}(t)$ denotes the proportion of the population infectious in the epidemic $E_{n}$ at time $t$. Therefore, the epidemic process corresponds to infectious individuals having an independent, exponentially distributed infectious period with parameter $\gamma$. Whilst infectious, an individual, $i$ say, makes global contacts at the points of a homogeneous Poisson point process with rate $\lambda_{\mathrm{G}}$, the individual contacted being chosen uniformly at random from the whole population, and makes local infectious contacts at the points of a homogeneous Poisson point process with rate $\lambda_{\mathrm{L}}$, the individual contacted being chosen according to $(i+V)_{\bmod n}$.

\section{Susceptibility process}

In the analysis of two-level mixing SIR epidemic models infectious clumps and susceptibility sets have proved useful; see [3] and [4]. We define the natural SIS epidemic analogue to the susceptibility set, which we will term the susceptibility process. The susceptibility process in 
itself is not particularly illuminating and we will therefore define a simpler quantity called the susceptibility time which will often prove sufficient for our needs.

For the contact process $C$, the susceptibility process is defined as follows. For $i, j \in \mathbb{Z}$ and $s \leq t$, we say that $i_{s} \rightsquigarrow j_{t}$ if there exists a path of local infection from individual $i$ at time $s$ to individual $j$ at time $t$. That is, if individual $i$ is infectious at time $s$ then individual $j$ will be infectious at time $t$ regardless of global infections. Therefore, the local infectious paths are determined by $\left\{\left(\eta_{i}^{\mathrm{R}}, \eta_{i}^{\mathrm{L}}, \boldsymbol{V}_{i}\right)\right\}_{i \in \mathbb{Z}}$, where $\boldsymbol{V}_{i}=\left(V_{i j}\right)_{j \in \mathbb{Z}}$. Then the susceptibility process of individual $i$ at time $t$ is given by

$$
\delta_{i}^{t}=\left\{S_{i}^{t, s} ; s \leq t\right\},
$$

where

$$
S_{i}^{t, s}=\left|\left\{j \in \mathbb{Z} ; j_{s} \rightsquigarrow i_{t}\right\}\right| .
$$

Thus, for $i \in \mathbb{Z}$ and $s \leq t, S_{i}^{t, s}$ is the total number of individuals who need to avoid global infection at time $s$ for individual $i$ to be susceptible at time $t$. The susceptibility time of individual $i$ at time $t$ is defined to be

$$
T_{i}^{t}=\int_{-\infty}^{t} S_{i}^{t, s} \mathrm{~d} s
$$

the total amount of time during which members of the population need to avoid global infectious contacts for individual $i$ to be susceptible at time $t$. The local infectious paths are spatially and temporally homogeneous. Therefore, for all $i \in \mathbb{Z}$ and $t \in \mathbb{R}, T_{i}^{t} \stackrel{D}{=} T_{0}^{0} \equiv T$, say, where 'D $=$ denotes equality in distribution. Given $s_{i}^{t}$ and $x(\cdot)$, the probability that individual $i$ is susceptible at time $t$ is

$$
\exp \left(-\lambda_{\mathrm{G}} \int_{-\infty}^{t} x(s) S_{i}^{t, s} \mathrm{~d} s\right)
$$

Thus, if $x(s)=u(s \in \mathbb{R})$ for some $u>0$ then (3.1) simplifies to

$$
\exp \left(-\lambda_{\mathrm{G}} u T_{i}^{t}\right)
$$

The above is directly applicable in scenario 1, where the initial configuration is constructed from the time lines. For scenario 2, we discuss any complications at time 0 in the susceptibility process and susceptibility time caused by specifying $\chi$ as they arise. Finally, note that the susceptibility process and susceptibility time are independent of the global infectious process.

For $n \geq 1$ and $1 \leq i \leq n$, the susceptibility process $\delta_{i, n}^{t}$ and susceptibility time $T_{i, n}^{t}$ can be defined in the obvious fashion for $C_{n}$ and $E_{n}$. Note that $C_{n}$ and $E_{n}$ only differ in the global infectious process, and so, can therefore be coupled to have identical susceptibility processes.

\section{Contact processes}

The aim of this section is to study contact processes with $\lambda_{\mathrm{G}}>0$ (global infection). The emphasis is upon the asymptotic behaviour of $\xi(t)$ as $t \rightarrow \infty$. Suppose that $x(t) \rightarrow u$ as $t \rightarrow \infty$ for some $u>0$. Then Lemma 4.1, below, shows that

$$
\xi(t) \stackrel{\mathrm{D}}{\rightarrow} \pi^{u} \quad \text { as } t \rightarrow \infty,
$$

where $\stackrel{\text { D }}{\rightarrow}$ ' denotes convergence in distribution and $\pi^{u}$ is an invariant measure on $\{0,1\}^{\mathbb{Z}}$. A realization from $\pi^{u}$ can be obtained from the time lines in Section 2 by fixing $x(s)=u(s \in \mathbb{R})$ and observing the status of the population at any fixed time $t$. Thus, $\pi^{u}$ is not a product measure 
and is generally hard to express. However, for $u>0, \mathrm{P}\left(\pi_{0}^{u}=1\right)=1-\mathrm{E}\left[\exp \left(-\lambda_{\mathrm{G}} u T\right)\right]$, where $T$ is the susceptibility time of a typical individual. Thus, exposing the population to $u$ units of global infectious pressure leads to a proportion $m(u)=1-\mathrm{E}\left[\exp \left(-\lambda_{\mathrm{G}} u T\right)\right]$ of the population being infectious. From an epidemic perspective we are interested in the solutions of the key equation

$$
u=m(u)\left(=1-\mathrm{E}\left[\exp \left(-\lambda_{\mathrm{G}} u T\right)\right]\right),
$$

that is, when are the infectious pressure and the proportion infectious equal? Clearly, $u=0$ is a solution of (4.1). Since $m(u)$ is a concave function, a solution $u^{*} \in(0,1]$ exists if and only if $R_{*}\left(=m^{\prime}(0)\right)=\lambda_{\mathrm{G}} \mathrm{E}[T]>1$. Note that $T$ depends upon $\lambda_{\mathrm{L}}$ and $V$, and so, $R_{*}$ and $u^{*}$ are functions of $\lambda_{\mathrm{G}}, \lambda_{\mathrm{L}}$, and $V$.

In the epidemic context the global infectious pressure depends upon the proportion of infectives. We therefore introduce self-referential infectious processes which seek to replicate this behaviour. However, since we have an infinite population, we define a sequence of selfreferential infectious processes, $\boldsymbol{y}_{n}=\left\{y_{n}(s) ; s \geq 0\right\}$, as follows. For $\boldsymbol{x}=\{x(s) ; s \geq 0\}$, let $\theta_{i}(t ; \boldsymbol{x}, \boldsymbol{\chi})=1$ and $\theta_{i}^{n}\left(t ; \boldsymbol{x}, \chi^{n}\right)=1$ if individual $i$ is infectious at time $t$ given that each individual is exposed to global infectious pressure at rate $\lambda_{\mathrm{G}} x(s)$ in $C$ and, respectively, in $C_{n}$ with $\theta_{i}(t ; \boldsymbol{x}, \boldsymbol{\chi})=0$ and, respectively, $\theta_{i}^{n}\left(t ; \boldsymbol{x}, \chi^{n}\right)=0$ otherwise. The $\left\{\theta_{i}^{n}\left(t ; \boldsymbol{x}, \boldsymbol{\chi}^{n}\right)\right\}$ are not required until Section 5 . Let $y_{n}(0)=(1 / n) \sum_{i=1}^{n} \mathrm{E}\left[\chi_{i}\right]$ and, for $t \geq 0$, let

$$
y_{n}(t)=\frac{1}{n} \sum_{i=1}^{n} \mathrm{E}\left[\theta_{i}\left(t ; \boldsymbol{y}_{n}, \boldsymbol{\chi}\right)\right] .
$$

Thus, $y_{n}(t)$ is the mean proportion of infectious individuals in $\{1,2, \ldots, n\}$ at time $t$ when the global infectious pressure at time $s$ is given by $y_{n}(s)$. Let $y(t)=\lim _{n \rightarrow \infty} y_{n}(t)$ should the limit exist. For $y(t)(t \geq 0)$ to exist and be nonzero, we require that $\chi$ is sufficiently regular in its behaviour and any of the following suffice:

(i) scenario 1 , where $\chi$ is given by the (past) time lines;

(ii) there exists $0<\phi \leq 1$ such that the components of $\chi$ are independent and identically distributed with $\mathrm{P}\left(\chi_{0}=1\right)=\phi$;

(iii) there exists $k \in \mathbb{N}$ such that, for all $m \in \mathbb{Z}$,

$$
\left(\chi_{m k+1}, \chi_{m k+2}, \ldots, \chi_{(m+1) k}\right)=\left(\chi_{1}, \chi_{2}, \ldots, \chi_{k}\right)
$$

with $\sum_{i=1}^{k} \chi_{i} \neq 0$.

Throughout, we will use $y(\cdot)$ to denote the self-referential process and $x(\cdot)$ to denote a generic global infectious process.

Theorem 4.1, below, states the main results of this section for self-referential processes. For simplicity in exposition, we assume that $y(t)$ exists with $y(0)=\phi>0$ and give the proofs in terms of the limit. However, all results hold for any $n \geq 1$ with $y_{n}(0)>0$. The key statement from Theorem 4.1 is that if $R_{*}>1$ and there is initially infinitely many infectives then, as $t \rightarrow \infty$, the proportion of the population infectious converges to $u^{*}$ and is distributed according to $\pi^{u^{*}}$. If $R_{*} \leq 1$, the proofs can be adapted to show that $\boldsymbol{\xi}(t) \stackrel{\mathrm{P}}{\rightarrow} \mathbf{0}$ as $t \rightarrow \infty$, a disease free equilibrium (where $\stackrel{\text { ' }}{\rightarrow}$ ' denotes convergence in probability). 
Theorem 4.1. Suppose that $R_{*}>1$ and that $u^{*}$ is the nonzero solution of (4.1). Then, for any $\chi$ such that $(1 / n) \sum_{i=1}^{n} \mathrm{E}\left[\chi_{i}\right] \rightarrow y(0) \equiv \phi>0$ as $n \rightarrow \infty$,

$$
y(t) \rightarrow u^{*} \text { as } t \rightarrow \infty .
$$

Moreover (by Lemma 4.1, below),

$$
\xi(t) \stackrel{\mathrm{D}}{\rightarrow} \pi^{u^{*}} \text { as } t \rightarrow \infty .
$$

Before proving Theorem 4.1, we give the necessary preliminary results. In the proceeding arguments, until otherwise stated, we assume a general global infectious process $x(\cdot)$.

A number of the key arguments throughout this paper rely upon establishing a coupling between two contact (epidemic) processes. Throughout, when comparing two contact (epidemic) processes, labelled process 1 and process 2 , respectively, we will index any quantities which differ between the two processes by the index of the appropriate process. Any quantities which are not indexed will be assumed to be the same for both processes.

Lemma 4.1. Suppose that there exists $u>0$ such that $x(t) \rightarrow u$ as $t \rightarrow \infty$. Then, for any $\chi \in\{0,1\}^{\mathbb{Z}}$ with $\boldsymbol{\xi}(0)=\chi$,

$$
\xi(t) \stackrel{\mathrm{D}}{\rightarrow} \pi^{u} \text { as } t \rightarrow \infty .
$$

Proof. By [7, p. 19], (4.5) holds if, for any $A \subset \mathbb{Z},|A|<\infty$,

$$
\boldsymbol{\xi}_{A}(t) \stackrel{\mathrm{D}}{\rightarrow} \pi_{A}^{u} \quad \text { as } t \rightarrow \infty,
$$

where $\xi_{A}(t)=\left(\xi_{i}(t)\right)_{i \in A}$ and $\pi_{A}^{u}=\left(\pi_{i}^{u}\right)_{i \in A}$.

Consider two contact processes with $\xi^{(1)}(0)=\chi$ and $\xi^{(2)} \stackrel{\mathrm{D}}{=} \pi^{u}$ and, for $s \geq 0, x_{1}(s)=x(s)$ and $x_{2}(s)=u$. The contact processes can be coupled using the same time lines and, hence, the same susceptibility processes. Thus, for an individual $i$, say, at time $t$,

$$
\mathrm{P}\left(\xi_{i}^{(1)}(t) \neq \xi_{i}^{(2)}(t)\right) \leq\left|\mathrm{P}\left(\xi_{i}^{(1)}(t)=0\right)-\mathrm{P}\left(\xi_{i}^{(2)}(t)=0\right)\right| .
$$

For $s \geq 0$, let

$$
\varepsilon_{s}=\sup _{w \geq s}|x(w)-u| .
$$

Then $\varepsilon_{s} \rightarrow 0$ as $s \rightarrow \infty$. To study (4.7), it is easiest to consider $T_{i}^{t} \leq t / 2$ and $T_{i}^{t}>t / 2$ separately. Since, for $s \geq t / 2, x(s) \geq u-\varepsilon_{t / 2}$, we have

$$
\begin{aligned}
\mathrm{P}\left(\xi_{i}^{(1)}(t)\right. & \left.\neq \xi_{i}^{(2)}(t)\right) \\
\leq & \exp \left(-\lambda_{\mathrm{G}}\left(u-\varepsilon_{t / 2}\right) \frac{t}{2}\right) \\
+ & \left|\mathrm{E}\left[\left(\exp \left(-\lambda_{\mathrm{G}} \int_{t / 2}^{t} x(s) S_{i}^{t, s} \mathrm{~d} s\right)-\exp \left(-\lambda_{\mathrm{G}} \int_{t / 2}^{t} u S_{i}^{t, s} \mathrm{~d} s\right)\right) \mid T_{i}^{t} \leq \frac{t}{2}\right]\right| .
\end{aligned}
$$

The latter term on the right-hand side of (4.8) is less than

$$
\begin{aligned}
& \mathrm{E}\left[\left(\exp \left(-\lambda_{\mathrm{G}}\left(u-\varepsilon_{t / 2}\right) T_{i}^{t}\right)-\exp \left(-\lambda_{\mathrm{G}}\left(u+\varepsilon_{t / 2}\right) T_{i}^{t}\right)\right) \mid T_{i}^{t} \leq \frac{t}{2}\right] \\
& \quad \leq \mathrm{E}\left[2 \lambda_{\mathrm{G}} \varepsilon_{t / 2} T_{i}^{t} \exp \left(-\lambda_{\mathrm{G}}\left(u-\varepsilon_{t / 2}\right) T_{i}^{t}\right) \mid T_{i}^{t} \leq \frac{t}{2}\right] \\
& \quad \rightarrow 0 \text { as } n \rightarrow \infty .
\end{aligned}
$$


Therefore, from (4.7), (4.8), and (4.9),

$$
\mathrm{P}\left(\xi_{i}^{(1)}(t) \neq \xi_{i}^{(2)}(t)\right) \rightarrow 0 \quad \text { as } n \rightarrow \infty .
$$

Since (4.10) holds for all $i$ and $\xi^{(2)}(t) \stackrel{\mathrm{D}}{=} \pi^{u}$ for all $t \in \mathbb{R}$, (4.6) follows immediately from (4.10).

The following lemma gives a useful coupling relationship which is used extensively in the proceeding arguments. Corollary 4.1, below, is an immediate consequence of Lemma 4.2.

Lemma 4.2. Suppose that, for contact processes 1 and $2, \lambda_{\mathrm{L}}^{1} \leq \lambda_{\mathrm{L}}^{2}, \lambda_{\mathrm{G}}^{1} \leq \lambda_{\mathrm{G}}^{2}, \chi_{(1)} \leq \chi_{(2)}$, and $x_{1}(s) \leq x_{2}(s)(s \geq 0)$. Then, for any finite subset $A$, provided that any of the above inequalities are strict,

$$
\frac{1}{|A|} \sum_{i \in A} \mathrm{E}\left[\xi_{i}^{(1)}(t)\right]<\frac{1}{|A|} \sum_{i \in A} \mathrm{E}\left[\xi_{i}^{(2)}(t)\right] .
$$

For (4.11), it is sufficient that there exists $a>0$ such that $x_{1}(b)<x_{2}(b)(0 \leq b<a)$ and $x_{1}(b) \leq x_{2}(b)(b \geq a)$.

Proof. The proof is similar to [14, Proposition 4.1] and, hence, the details are omitted.

Corollary 4.1. Let $x(\cdot)$ be nonincreasing or nondecreasing in $t \geq 0$. Suppose that there exists $a \geq 0$ such that, for all $h>0, x(a+h)<x(a)(x(a+h)>x(a))$. Then given scenario 1 , where $\chi$ is constructed from the (past) time lines, for $i \in \mathbb{Z}$,

$$
\mathrm{P}\left(\xi_{i}(t)=1 \mid\{x(s) ; s \geq 0\}\right)
$$

is strictly decreasing or, respectively, increasing in $t$.

We now devote ourselves to the self-referential process. We begin by considering the special case where $\chi \stackrel{\mathrm{D}}{=} \pi^{u}(u>0)$. This is scenario 1 with $x(s)=u(s<0)$. For $u>0$, let

$$
\hat{y}_{u}(t)=1-\mathrm{E}\left[\exp \left(-\lambda_{\mathrm{G}} \int_{-\infty}^{t} \hat{y}_{u}(s) S_{0}^{t, s} \mathrm{~d} s\right)\right],
$$

where $\hat{y}_{u}(s)=u(s<0)$. Since the epidemic generated by scenario 1 is spatially homogeneous, $\hat{y}_{u}(t)=\mathrm{E}\left[\xi_{0}(t)\right]$ and, for all $n \geq 1, y_{n}(t)=\hat{y}_{u}(t)$.

Lemma 4.3. Suppose that $\xi(0) \stackrel{\mathrm{D}}{=} \pi^{u}$ for some $0<u \leq \infty$. Then

$$
\hat{y}_{u}(t) \rightarrow u^{*} \text { as } t \rightarrow \infty
$$

where $u^{*}$ is the nonzero solution of (4.1).

Proof. The lemma is immediate if $u=u^{*}$. We prove the result for $u>u^{*}$ with similar arguments applying for $u<u^{*}$.

Fix $u_{0}=u>u^{*}$, and let $\tilde{u}_{0}=m_{u_{0}}$. Since $u_{0}>u^{*}, u^{*}<\tilde{u}_{0}<u_{0}$. Let $\tilde{u}_{0}<u_{1}<u_{0}$ with $\tilde{u}_{1}=m_{u_{1}}$. Then, for $k=2,3, \ldots$, let $u_{k}$ satisfy $\tilde{u}_{k-1}<u_{k}<\tilde{u}_{k-2}$, where $\tilde{u}_{k}=m_{u_{k}}$. Since $m_{u}$ is concave in $u, u_{k}, \tilde{u}_{k} \rightarrow u^{*}$ as $k \rightarrow \infty$.

Let $0=\tilde{t}_{0}<\tilde{t}_{1}<\cdots$ be a sequence of times to be defined shortly. For $k=1,2, \ldots$ and $t>0$, let $\psi^{k}(t)=\left(\ldots, \psi_{-1}^{k}(t), \psi_{0}^{k}(t), \psi_{1}^{k}(t), \ldots\right)$ denote the state of the population at time $t$ in the contact process $\tilde{C}_{k}$ with piecewise-constant global infectious pressure, $\boldsymbol{\psi}^{k}(0)=\boldsymbol{\xi}(0)\left(\stackrel{\mathrm{D}}{=} \pi^{u}\right)$ 
and $\psi_{i}^{k}(t)=1$ if individual $i$ is infectious at time $t$ and $\psi_{i}^{k}(t)=0$ otherwise. Specifically, let $x_{k}(\cdot)$ denote the global infectious pressure in $\tilde{C}_{k}$ with

$$
x_{k}(t)= \begin{cases}u_{0}, & t<0 \\ u_{l}, & \tilde{t}_{l-1} \leq t<\tilde{t}_{l}, l=1,2, \ldots, k-1, \\ u_{k}, & t>\tilde{t}_{k-1} .\end{cases}
$$

For $k=1,2, \ldots$ and $t \geq 0$, let $z_{k}(t)=\mathrm{E}\left[\psi_{0}^{k}(t)\right]$. Then $z_{k}(t) \rightarrow \tilde{u}_{k}$ and $\boldsymbol{\psi}^{k}(t) \stackrel{\mathrm{D}}{\rightarrow} \boldsymbol{\pi}^{u_{k}}$ as $t \rightarrow \infty$.

Note that $\pi^{u^{*}}<_{\mathrm{st}} \boldsymbol{\pi}^{u} \stackrel{\mathrm{D}}{=} \boldsymbol{\xi}(0)$, where ' $<_{\mathrm{st}}$ ' denotes 'stochastically smaller than'. Therefore, by Lemma 4.2 , for all $t \geq 0$,

$$
\liminf _{t \rightarrow \infty} \hat{y}_{u}(t) \geq \liminf _{t \rightarrow \infty} \hat{y}_{u^{*}}(t)=u^{*} .
$$

The main part of the proof involves showing that, for all $t \geq 0$ and $k=1,2, \ldots$,

$$
\hat{y}_{u}(t) \leq z_{k}(t)
$$

and, hence, $\lim \sup _{t \rightarrow \infty} \hat{y}_{u}(t) \leq u^{*}$, from which the lemma follows immediately.

Firstly, we define $\tilde{\boldsymbol{t}}=\left(\tilde{t}_{0}, \tilde{t}_{1}, \ldots\right)$. For $k=1,2, \ldots$, let $\tilde{t}_{k}$ be such that $z_{k}\left(\tilde{t}_{k}\right)=u_{k+1}$. Then, for all $t \geq \tilde{t}_{k}, z_{k}(t)<u_{k+1}$. Note that $\hat{y}_{u}(0)=\tilde{u}_{0}<u_{1}$. Therefore, since $\hat{y}_{u}(\cdot)$ is continuous, there exists $t_{1}^{*}$ such that, for all $0 \leq t \leq t_{1}^{*}, \hat{y}_{u}(t)<u_{1}=x_{1}(t)$. Hence, for all $0 \leq t \leq t_{1}^{*}$,

$$
\hat{y}_{u}(t)<z_{1}(t) \text {. }
$$

However, by Corollary 4.1, $z_{1}(t)$ is decreasing with $z_{1}(t) \leq \tilde{u}_{0}<u(t \geq 0)$, and so, it is straightforward to show that (4.14) holds for all $t \geq 0$.

Fix $k>1$. Suppose that, for all $t>0$,

$$
\hat{y}_{u}(t)<z_{k-1}(t)
$$

Then, for all $0 \leq t<\tilde{t}_{k-1}$,

$$
\hat{y}_{u}(t)<z_{k-1}(t) \equiv z_{k}(t) .
$$

Therefore, $\hat{y}_{u}\left(\tilde{t}_{k-1}\right)<u_{k}$, and so, there exists $t_{k}^{*}>\tilde{t}_{k-1}$ such that, for all $\tilde{t}_{k-1} \leq t<t_{k}^{*}, x(t)<$ $u_{k}$. Hence, for all $0 \leq t \leq t_{k}^{*}$,

$$
\hat{y}_{u}(t)<z_{k}(t)
$$

Since, by Corollary 4.1, $z_{k}(t)$ is decreasing in $t$ with $z_{k}(t) \leq \tilde{u}_{k-1}\left(t \geq \tilde{t}_{k-1}\right)$, it follows that (4.15) holds for all $t>0$.

Therefore, since $z_{k}(t) \rightarrow \tilde{u}_{k}$ as $t \rightarrow \infty$, for any $\varepsilon>0$, there exists $K \in \mathbb{N}$ such that $\tilde{u}_{K}<u^{*}+\varepsilon$, and so,

$$
\limsup _{t \rightarrow \infty} \hat{y}_{u}(t) \leq \limsup _{t \rightarrow \infty} z_{K}(t)=\tilde{u}_{K}<u^{*}+\varepsilon .
$$

The lemma follows from (4.13) and (4.16).

For $\boldsymbol{\xi}(0) \stackrel{\mathrm{D}}{=} \pi^{u}(0<u \leq \infty), \boldsymbol{\xi}(t)$ and $\hat{y}_{u}(t)$ are either (stochastically) increasing or decreasing in $t$. This is not the case for general $\xi(0) \stackrel{\mathrm{D}}{=} \chi$ and needs to be addressed in order to conclude the proof of Theorem 4.1 . 
Proof of Theorem 4.1. Clearly, $\chi \leq_{\mathrm{st}} \pi^{\infty}$, where $\pi^{\infty}$ corresponds to everybody being initially infectious. Thus, it is immediate from Lemmas 4.1 and 4.2 that $\lim _{\sup _{t \rightarrow \infty}} y(t)=u^{*}$. Similarly, if there exists $u>0$ such that $\pi^{u} \leq_{\mathrm{st}} \chi$ then $\liminf _{t \rightarrow \infty} y(t)=u^{*}$.

More generally, a lower bound for $y(t)$ can be obtained by noting that, for all $t \geq 0$, $y(t) \geq \phi \mathrm{e}^{-t}$, since $\mathrm{e}^{-t}$ is the probability an initially infectious individual has not recovered by time $t$. Thus, a sequence of lower bound processes in the spirit of Lemma 4.1 can be constructed as follows.

For $v \geq 0$, let $\hat{T}_{v}=\int_{-v}^{0} S_{0}^{0, s} \mathrm{~d} s$. Then $\hat{T}_{v} \stackrel{\mathrm{D}}{\rightarrow} T$ as $v \rightarrow \infty$ and, therefore, there exists $\kappa^{*}>0$ such that, for all $v>\kappa^{*}, \lambda_{\mathrm{G}} \mathrm{E}\left[\hat{T}_{\nu}\right]>1$. Fix $\kappa>\kappa^{*}$. Then $1-\mathrm{E}\left[\exp \left(-\lambda_{\mathrm{G}} t \hat{T}_{\kappa}\right)\right]$ is concave in $t$, and so, there exists $\delta^{*}>0$ such that, for all $0<\tilde{\delta}<\delta^{*}$,

$$
1-\mathrm{E}\left[\exp \left(-\lambda_{\mathrm{G}} \tilde{\delta} \hat{T}_{\kappa}\right)\right]>\tilde{\delta} .
$$

Fix $0<\delta<\min \left\{\delta^{*}, \phi \mathrm{e}^{-\kappa}\right\}$. Consider a lower bound process $\boldsymbol{\psi}^{1}(t)$. Let

$$
x_{1}(s)= \begin{cases}0, & s<0 \\ \delta, & s \geq 0\end{cases}
$$

denote the global infectious pressure that individuals in $\psi^{1}(t)$ are subjected to at time $s$ with $\boldsymbol{\psi}^{1}(0)=\mathbf{0}$. Let $z_{1}(t)=\mathrm{E}\left[\psi_{0}^{1}(t)\right]$. For all $0 \leq t \leq \kappa$, by Lemma 4.2,

$$
z_{1}(t)<y(t)
$$

since $x(t) \geq \delta$. Moreover, by Corollary 4.1, z(.) is increasing with

$$
\delta<z(\kappa)<y(\kappa)
$$

Thus, a new lower bound process $\psi^{2}(t)$ can be constructed with global infectious pressure $x_{2}(t)$, where

$$
x_{2}(t)= \begin{cases}x_{1}(t), & t \leq \kappa, \\ z_{1}(\kappa), & t>\kappa ;\end{cases}
$$

cf. (4.12). This process can be repeated to form a sequence of lower bounds as in Lemma 4.3. Thus, it can be shown that

$$
\liminf _{t \rightarrow \infty} y(t)=u^{*}
$$

Therefore, (4.3) is proved and (4.4) follows by Lemma 4.1.

\section{Convergence of epidemic processes}

The reason for introducing the contact process was to represent the limiting behaviour of the sequence of epidemic processes $\left(E_{n}\right)$ as $n \rightarrow \infty$. From the descriptions of the epidemic and contact processes upon $\{1,2, \ldots, n\}$ given in Section 2 , the epidemic process is the special case of the contact process, where the global infectious pressure depends upon the (stochastic) proportion of the population infectious.

The statement of the main result follows the proceeding observation. For $n \geq 1$ and $t \geq 0$, let $\chi^{n}=\left(\chi_{1}, \chi_{2}, \ldots, \chi_{n}\right)$ and $Y_{n}\left(t ; \chi^{n}\right)$ denote the initial configuration of infectives and the proportion of the population infectious at time $t$, respectively, in the epidemic $E_{n}$. 
Let $\theta_{i}^{n}\left(t ; \boldsymbol{Y}_{n}, \chi^{n}\right)$ be defined as in Section 4 . Then $\theta_{i}^{n}\left(t ; \boldsymbol{Y}_{n}, \boldsymbol{\chi}^{n}\right)=1$ if individual $i$ is infectious at time $t$ in $E_{n}$ and

$$
Y_{n}\left(t ; \chi^{n}\right)=\frac{1}{n} \sum_{i=1}^{n} \theta_{i}^{n}\left(t ; \boldsymbol{Y}_{n}, \chi^{n}\right)
$$

cf. [14, Section 2].

Theorem 5.1. For $t \geq 0$, let $y_{n}(t)$ be defined by (4.2). Then

$$
\sup _{0 \leq s \leq t}\left|Y_{n}\left(s ; \chi^{n}\right)-y_{n}(s)\right| \stackrel{\mathrm{P}}{\rightarrow} 0 \quad \text { as } n \rightarrow \infty .
$$

Note that if, for all $t \geq 0, y(t)=\lim _{n \rightarrow \infty} y_{n}(t)$ exists then $y_{n}(s)$ can be replaced in (5.1) by $y(s)$. As mentioned in Section 4 , this will be the case if $\chi$ is sufficiently well behaved and sufficient conditions are presented in Section 4. Theorem 5.1 is a functional law of large numbers which states that the solution of (4.2) (the deterministic (mean) proportion infectious) provides a good approximation for the (stochastic) proportion infectious in $E_{n}$ over any finite time interval. The main results proved in Section 4 are asymptotic as $t \rightarrow \infty$ and it can easily be shown that, for any $n \geq 1, Y_{n}\left(t ; \chi^{n}\right) \stackrel{\mathrm{P}}{\rightarrow} 0$ as $t \rightarrow \infty$. However, for large $t,\left\{Y_{n}\left(t ; \chi^{n}\right) \mid Y_{n}\left(t ; \chi^{n}\right)>0\right\} \approx u^{*}$.

Theorem 5.1 will be proved using the triangle inequality via a series of lemmas. We begin by introducing a new process $\left\{Y_{n}^{1}(\cdot ; \cdot)\right\}$ which is easier to analyse. For $n \geq 1$, let

$$
Y_{n}^{1}(t ; \chi)=\frac{1}{n} \sum_{i=1}^{n} \theta_{i}\left(t ; \boldsymbol{Y}_{n}, \chi\right) .
$$

Since $\chi^{n}=\left(\chi_{1}, \chi_{2}, \ldots, \chi_{n}\right), Y_{n}\left(t ; \chi^{n}\right)$ and $Y_{n}^{1}\left(t ; \chi^{n}\right)$ can be coupled on a common probability space such that $Y_{n}\left(0 ; \chi^{n}\right) \equiv Y_{n}^{1}\left(0 ; \chi^{n}\right)$ and $\theta_{i}\left(t ; \boldsymbol{Y}_{n}, \boldsymbol{\chi}\right) \neq \theta_{i}^{n}\left(t ; \boldsymbol{Y}_{n}, \boldsymbol{\chi}^{n}\right)$ only if individual $i$ 's susceptibility processes differs between $E_{n}$ and $C$ on the interval $[0, t]$. Therefore, we can construct bounds for

$$
\mathrm{P}\left(\theta_{i}\left(t ; \boldsymbol{Y}_{n}, \boldsymbol{\chi}\right) \neq \theta_{i}^{n}\left(t ; \boldsymbol{Y}_{n}, \boldsymbol{\chi}^{n}\right)\right) .
$$

Let $\tilde{\mathrm{R}}_{t}$ denote the right-most infective at time $t$ in the following simple $\mathrm{S} \rightarrow$ I epidemic model amongst a population located at the points of $\mathbb{Z}$. At time 0 , individuals . ., $-2,-1,0$ are infectious whilst individuals $1,2, \ldots$ are susceptible. Similarly, let $-\tilde{\mathrm{L}}_{t}$ denote the left-most infective at time $t$ in an $\mathrm{S} \rightarrow \mathrm{I}$ epidemic where at time 0 , individuals $0,1,2, \ldots$ are infectious and individuals $\ldots,-2,-1$ are susceptible. Individuals, if infectious, make (local) infectious contacts at the points of a homogeneous Poisson point process with rate $\lambda_{\mathrm{L}}$ with the individual contacted distributed according to $i+V$. Then

$$
\tilde{\mathrm{R}}_{t} \leq \mathrm{st} \sum_{j=1}^{\tilde{A}_{t}} \tilde{B}_{j},
$$

where $\tilde{A}_{t} \sim \operatorname{Po}\left(\lambda_{\mathrm{L}} \mathrm{E}[|V|] t / 2\right)$ and $\tilde{B}_{1}, \tilde{B}_{2}, \ldots$ are independent and identically distributed according to $\tilde{B}$ with $\mathrm{P}(\tilde{B}=k)=2 \mathrm{P}(V \geq k) / \mathrm{E}[|V|]$.

Lemma 5.1. For any $t>0$,

$$
\mathrm{P}\left(\tilde{\mathrm{R}}_{t} \geq k\right) \leq \frac{1}{k} \mathrm{E}[\tilde{B}] \mathrm{E}\left[\tilde{A}_{t}\right],
$$

where $\mathrm{E}[\tilde{B}]<\infty$ and $\mathrm{E}\left[\tilde{A}_{t}\right]=\lambda_{\mathrm{L}} \mathrm{E}[|V|] t / 2$.

Since $V$ is symmetric, (5.2) holds with $\tilde{\mathrm{L}}_{t}$ in place of $\tilde{\mathrm{R}}_{t}$. 
Proof. The proof is straightforward using Markov's inequality.

The following corollary is an immediate consequence of Lemma 5.1.

Corollary 5.1. For all $t \geq 0$,

$$
\sup _{0 \leq s \leq t}\left|Y_{n}\left(s ; \chi^{n}\right)-Y_{n}^{1}(s ; \chi)\right| \stackrel{\mathrm{P}}{\rightarrow} 0 \quad \text { as } n \rightarrow \infty .
$$

Proof. Fix $\varepsilon>0$. Since, for $t \geq 0, \tilde{\mathrm{R}}_{t}=\sup _{0 \leq s \leq t} \tilde{\mathrm{R}}_{s}$, we have, by Markov's inequality and Lemma 5.1,

$$
\begin{aligned}
& \mathrm{P}\left(\sup _{0 \leq s \leq t}\left|Y_{n}\left(s ; \chi^{n}\right)-Y_{n}^{1}(s ; \chi)\right|>\varepsilon\right) \\
& \quad \leq \frac{1}{\varepsilon n} \sum_{i=1}^{n} \mathrm{E}\left[\sup _{0 \leq s \leq t}\left|\theta_{i}^{n}\left(s ; \boldsymbol{Y}_{n}, \chi^{n}\right)-\theta_{i}\left(s ; \boldsymbol{Y}_{n}, \chi\right)\right|\right] \\
& \quad \leq \frac{1}{\varepsilon n} \sum_{i=1}^{n}\left(\mathrm{P}\left(\tilde{\mathrm{R}}_{t} \geq i\right)+\mathrm{P}\left(\tilde{\mathrm{L}}_{t} \geq n+1-i\right)\right) \\
& \quad \rightarrow 0 \quad \text { as } n \rightarrow \infty .
\end{aligned}
$$

Therefore, analysis can be focused upon $Y_{n}^{1}(\cdot ; \cdot)$ rather than $Y_{n}(\cdot ; \cdot)$.

Lemma 5.2. For all $t \geq 0$,

$$
\sup _{0 \leq s \leq t}\left|Y_{n}^{1}(s ; \chi)-\mathrm{E}\left[Y_{n}^{1}(s ; \chi) \mid \boldsymbol{Y}_{n}\right]\right| \stackrel{\mathrm{P}}{\rightarrow} 0 \text { as } n \rightarrow \infty .
$$

Proof. Fix $t \geq 0$ and note that, for $0 \leq s \leq t$,

$$
Y_{n}^{1}(s ; \chi)-\mathrm{E}\left[Y_{n}^{1}(s ; \chi) \mid \boldsymbol{Y}_{n}\right]=\frac{1}{n} \sum_{i=1}^{n}\left(\theta_{i}\left(s ; \boldsymbol{Y}_{n}, \chi\right)-\mathrm{E}\left[\theta_{i}\left(s ; \boldsymbol{Y}_{n}, \boldsymbol{\chi}\right) \mid \boldsymbol{Y}_{n}\right]\right) .
$$

Using the theorem of total probability and Chebyshev's inequality (cf. [13, Lemma 5.4]),

$$
\begin{aligned}
\mathrm{P}\left(\left|\frac{1}{n} \sum_{i=1}^{n}\left\{\theta_{i}\left(s ; \boldsymbol{Y}_{n}, \boldsymbol{\chi}\right)-\mathrm{E}\left[\theta_{i}\left(s ; \boldsymbol{Y}_{n}, \boldsymbol{\chi}\right) \mid \boldsymbol{Y}_{n}\right]\right\}\right|>\varepsilon\right) \\
\quad \leq \frac{1}{\varepsilon^{2} n^{2}} \sum_{i=1}^{n} \sum_{j=1}^{n} \mathrm{E}\left[\operatorname{cov}\left(\theta_{i}\left(s ; \boldsymbol{Y}_{n}, \boldsymbol{\chi}\right), \theta_{j}\left(t ; \boldsymbol{Y}_{n}, \boldsymbol{\chi}\right) \mid \boldsymbol{Y}_{n}\right)\right]
\end{aligned}
$$

For $t \geq 0$ and $k \geq 0$, let $\hat{\mathrm{R}}_{i, k}(t)$ and $\hat{\mathrm{L}}_{j, k}(t)$ denote the furthest individual to the right and left, respectively, that is infected up to time $t$ by the local epidemic having as initial infectives all individuals less than or equal to $i+[k / 2]$ and greater than or equal to $j-[k / 2]+1$, respectively, where $[x]$ denotes the largest integer less than or equal to $x$. For $i<j$, let $B_{i, j}(s)=\left\{\hat{\mathrm{R}}_{i, j-i}(s) \geq j\right\} \cup\left\{\hat{\mathrm{L}}_{j, j-i}(t) \leq i\right\}$. Then following [4, Lemma 3.1], for any $\boldsymbol{y}$,

$$
\begin{aligned}
& \left|\operatorname{cov}\left(\theta_{i}(t ; \boldsymbol{x}, \boldsymbol{\chi}), \theta_{j}(t ; \boldsymbol{x}, \boldsymbol{\chi})\right)\right| \\
& \quad \leq\left|\mathrm{P}\left(\theta_{i}(t ; \boldsymbol{x}, \boldsymbol{\chi})=1, \theta_{j}(t ; \boldsymbol{x}, \boldsymbol{\chi})=1\right)-\mathrm{P}\left(\theta_{i}(t ; \boldsymbol{x}, \boldsymbol{\chi})=1\right) \mathrm{P}\left(\theta_{j}(t ; \boldsymbol{x}, \boldsymbol{\chi})=1\right)\right| \\
& \quad \leq 5 \mathrm{P}\left(B_{i, j}(t)\right) \\
& \quad \leq 5\left(\mathrm{P}\left(\tilde{\mathrm{R}}_{t}>\left[\frac{j-i}{2}\right]\right)+\mathrm{P}\left(\tilde{\mathrm{L}}_{t}>\left[\frac{j-i}{2}\right]\right)\right) .
\end{aligned}
$$


From Lemma 5.1, for $j-i \geq 2$,

$$
\operatorname{cov}\left(\theta_{i}(s ; \boldsymbol{x}, \chi), \theta_{j}(s ; \boldsymbol{x}, \chi)\right) \leq(5)(2) \frac{3}{j-i} \frac{\lambda_{\mathrm{L}} \mathrm{E}[|V|] s}{2} \mathrm{E}[\tilde{B}],
$$

and so, from (5.4) and (5.5),

$$
\begin{aligned}
\mathrm{P}\left(\mid \frac{1}{n}\right. & \left.\sum_{i=1}^{n}\left(\theta_{i}\left(s ; \boldsymbol{Y}_{n}, \boldsymbol{\chi}\right)-\mathrm{E}\left[\theta_{i}\left(s ; \boldsymbol{Y}_{n}, \boldsymbol{\chi}\right) \mid \boldsymbol{Y}_{n}\right]\right) \mid>\varepsilon\right) \\
& \leq \frac{1}{\varepsilon n^{2}} 2 n\left(2+15 \sum_{k=2}^{n} \frac{1}{k} \lambda_{\mathrm{L}} \mathrm{E}[|V|] s \mathrm{E}[\tilde{B}]\right) \\
& \rightarrow 0 \text { as } n \rightarrow \infty
\end{aligned}
$$

Finally, (5.3) follows from (5.6) along similar lines to the proof of [14, Lemma 3.2] and, hence, the details are omitted.

Before proving the final step of Theorem 5.1, we require the following result. Couple two epidemic (contact) processes with the same initial configuration $\chi$, local transitions, and removal times. That is, any given individual $i$ has the same susceptibility process in both epidemic (contact) processes. Let processes 1 and 2 be subjected to global infectious processes $x_{1}(\cdot)$ and $x_{2}(\cdot)$, respectively. For $j=1,2$, let $\tilde{\theta}_{i}^{j}\left(t ; \boldsymbol{x}_{j}, \boldsymbol{\chi}\right)=1$ if individual $i$ is infectious in process $j$ at time $t$ and $\tilde{\theta}_{i}^{j}\left(t ; \boldsymbol{x}_{j}, \boldsymbol{\chi}\right)=0$ otherwise. We then have the following lemma which is similar to [14, Proposition 3.1].

Lemma 5.3. There exists a coupling of the two epidemic (contact) processes described above such that, for all $i \in\{1,2, \ldots, n\}(i \in \mathbb{Z})$,

$$
\begin{aligned}
\mathrm{P}\left(\tilde{\theta}_{i}^{1}\left(t ; \boldsymbol{x}_{1}, \boldsymbol{\chi}\right) \neq \tilde{\theta}_{i}^{2}\left(t ; \boldsymbol{x}_{2}, \boldsymbol{\chi}\right)\right) & \leq \lambda_{\mathrm{G}} \int_{0}^{t}\left|x_{1}(s)-x_{2}(s)\right| \mathrm{E}\left[S_{i}^{t, s}\right] \mathrm{d} s \\
& \leq \lambda_{\mathrm{G}} \int_{0}^{t}\left|x_{1}(s)-x_{2}(s)\right| \exp \left(\lambda_{\mathrm{L}}(t-s)\right) \mathrm{d} s .
\end{aligned}
$$

Proof of Theorem 5.1. Note that

$$
\begin{aligned}
\sup _{0 \leq s \leq t}\left|\mathrm{E}\left[Y_{n}^{1}(s ; \boldsymbol{\chi}) \mid \boldsymbol{Y}_{n}\right]-y_{n}(s)\right| \\
\quad \leq \sup _{0 \leq s \leq t}\left|\frac{1}{n} \sum_{i=1}^{n} \mathrm{E}\left[\theta_{i}\left(s ; \boldsymbol{Y}_{n}, \boldsymbol{\chi}\right) \mid \boldsymbol{Y}_{n}\right]-\mathrm{E}\left[\theta_{i}\left(t ; \boldsymbol{y}_{n}, \boldsymbol{\chi}\right)\right]\right| \\
\quad \leq \sup _{0 \leq s \leq t} \frac{1}{n} \sum_{i=1}^{n}\left|\mathrm{E}\left[\theta_{i}\left(s ; \boldsymbol{Y}_{n}, \boldsymbol{\chi}\right) \mid \boldsymbol{Y}_{n}\right]-\mathrm{E}\left[\theta_{i}\left(t ; \boldsymbol{y}_{n}, \boldsymbol{\chi}\right)\right]\right| \\
\quad \leq \sup _{0 \leq s \leq t} \lambda_{\mathrm{G}} \int_{0}^{t}\left|Y_{n}(s)-y_{n}(s)\right| \exp \left(\lambda_{\mathrm{L}}(t-s)\right) \mathrm{d} s \\
\quad \leq \int_{0}^{t} \lambda_{\mathrm{G}} \exp \left(\lambda_{\mathrm{L}}(t-s)\right) \sup _{0 \leq u \leq s}\left|Y_{n}(u)-y_{n}(u)\right| \mathrm{d} s,
\end{aligned}
$$

where the penultimate inequality in (5.7) follows from Lemma 5.3. 
For $t \geq 0$, let

$$
A_{n}^{1}(t)=\sup _{0 \leq s \leq t}\left|Y_{n}\left(s ; \chi^{n}\right)-Y_{n}^{1}(s ; \chi)\right|
$$

and

$$
A_{n}^{2}(t)=\sup _{0 \leq s \leq t}\left|Y_{n}^{1}(s ; \chi)-\mathrm{E}\left[Y_{n}^{1}(s ; \chi) \mid \boldsymbol{Y}_{n}\right]\right| .
$$

Then, by the triangle inequality followed by Gronwall's inequality,

$$
\begin{aligned}
\sup _{0 \leq s \leq t} & \left|Y_{n}\left(s ; \chi^{n}\right)-y_{n}(s)\right| \\
& \leq A_{n}^{1}(t)+A_{n}^{2}(t)+\int_{0}^{t} \lambda_{\mathrm{G}} \exp \left(\lambda_{\mathrm{L}}(t-s)\right) \sup _{0 \leq u \leq s}\left|Y_{n}\left(u ; \chi^{n}\right)-y_{n}(u)\right| \mathrm{d} s \\
& \leq\left(A_{n}^{1}(t)+A_{n}^{2}(t)\right) \exp \left(\int_{0}^{t} \lambda_{\mathrm{G}} \exp \left(\lambda_{\mathrm{L}}(t-s)\right) \mathrm{d} s\right) \\
& \leq\left(A_{n}^{1}(t)+A_{n}^{2}(t)\right) \exp \left(\frac{\lambda_{\mathrm{G}}}{\lambda_{\mathrm{L}}}\left(\exp \left(\lambda_{\mathrm{L}} t\right)-1\right)\right) .
\end{aligned}
$$

From Corollary 5.1 and Lemma 5.2, respectively, $A_{n}^{1}(t) \stackrel{\mathrm{P}}{\rightarrow} 0$ and $A_{n}^{2}(t) \stackrel{\mathrm{P}}{\rightarrow} 0$ as $n \rightarrow \infty$. Therefore, the right-hand side of (5.8) converges in probability to 0 as $n \rightarrow \infty$ and the theorem is proved.

\section{Branching process approximation and invasion}

The results presented in Sections 4 and 5 are relevant if the initial number of infectives is large, namely, $(1 / n) \sum_{i=1}^{n} \chi_{i} \stackrel{\mathrm{P}}{\rightarrow} \phi$ as $n \rightarrow \infty$ for some $0<\phi \leq 1$. However, of general interest is the progression of the disease started from one initial infective, or more generally $a$ initial infectives $(1 \leq a<\infty)$. In this case the main scientific questions of interest are, can a major epidemic outbreak occur and what is the probability of a major epidemic? Answers are provided in Corollary 6.1, below, with a link established between the probability of a major outbreak and the proportion of the population infectious in endemic equilibrium.

The initial stages of both SIR and SIS epidemics can be modelled in a similar manner with branching process approximations being appropriate in both cases. A branching process approximation for the SIR great circle epidemic model is developed in detail in [4, Section 2], and can, with a little care, be adapted to the SIS great circle epidemic model presented above. Therefore, we give an outline of the approximating branching process and an alternative construction of the epidemic which is amenable for coupling to the branching process. Note that individuals in the branching process correspond to local epidemic clumps (see the description below) in the epidemic process.

Consider a sequence of independent and identically distributed contact processes $C_{k}(k=$ $1,2, \ldots)$ constructed as follows. For fixed $k, C_{k}$ is constructed using the time lines described in Section 2 with the only difference being global infections. Let $\eta^{\mathrm{G}}$ be a homogeneous Poisson point process with rate $\lambda_{\mathrm{G}}$ and, for individual $i$ in $C_{k}$, let $\eta_{k i}^{\mathrm{G}}$ be the points of time, if infectious, at which individual $i$ makes global infectious contacts. Thus, $\eta^{\mathrm{G}}$ denotes the (potential) global infectious contacts made by an individual as opposed to those received by an individual earlier in the paper. Initially at time 0 , individual 0 is assumed to be infectious in an otherwise susceptible population. The local epidemic clump is constructed from the time lines ignoring global infectious contacts. Let $D_{k}$ denote the duration of the epidemic $C_{k}$. For $t \geq 0$, let $J_{k}(t)$ denote the individuals whom have been infected in $C_{k}$ up to time $t$ with $\boldsymbol{J}_{k}=\left\{J_{k}(t) ; t \geq 0\right\}$. 
Let $I_{k}(t)$ denote the total number of infectives in $C_{i}$ at time $t$. Then global infectious contacts are made by the infectives in $C_{k}$ at the points of an inhomogeneous Poisson point process $\tilde{\eta}_{k}$ with rate $\lambda_{\mathrm{G}} I_{k}(t)$.

Similarly, for $n=1,2, \ldots$, local epidemic clumps $C_{1}^{n}, C_{2}^{n}, \ldots$ can be constructed on a circle. For fixed $n$ and $k$, label the individuals on the circle $-[(n-1) / 2],-[(n-1) / 2]+$ $1, \ldots,[n / 2]$. Assume that initially individual 0 is infectious in an otherwise susceptible population. Then $C_{k}^{n}$ can be coupled to $C_{k}$ with the only difference being that local infectious contacts by individual $i(-[(n-1) / 2] \leq i \leq[n / 2])$ are chosen according to $(i+V)_{\bmod n}$. Let $D_{k}^{n}, \tilde{\eta}_{k}^{n}, I_{k}^{n}(t), J_{k}^{n}(t)(t \geq 0)$, and $\boldsymbol{J}_{k}^{n}$ be defined in the obvious fashion.

A branching process can be constructed from the contact processes as follows. Let $0<$ $s_{2} \leq s_{3} \leq \cdots$ denote the times of births in the branching process, and let $\left(D_{2}, J_{2}, \tilde{\eta}_{2}\right)$, $\left(D_{3}, \boldsymbol{J}_{3}, \tilde{\eta}_{3}\right), \ldots$ denote the successive histories of individuals born into the branching process. (Set $s_{k+1}=\infty$ if fewer than $k$ individuals are born into the branching process.) Denote by $\left(D_{1}, \boldsymbol{J}_{1}, \tilde{\eta}_{1}\right)$ the history of the individual initially present in the branching process. Thus, individuals in the branching process are labelled in the order in which they are born, with label 1 being attached to the initial ancestor. The $k$ th individual in the branching process has lifetime $D_{k}$ and reproduces at the points of $\tilde{\eta}_{k}$. The $\boldsymbol{J}_{k}$ s play no role in the branching process, but are instrumental in coupling the epidemic process to the branching process. The above construction is similar to that given in [4, p. 237]. The only difference is in the explicit construction of $C_{k}$. Finally, let $X(t)$ denote the total number of individuals alive in the branching process at time $t$.

For $n=1,2, \ldots$, a realisation of the epidemic $E_{n}$ can be constructed as follows. Let $\chi_{k}^{n}$ $(k=1,2, \ldots)$ be independent random variables, each uniformly distributed on $\{1,2, \ldots, n\}$. The initial infective is the individual $\chi_{1}^{n}$ and the individual contacted at the $k$ th global contact made in $E_{n}$ is $\chi_{k+1}^{n}$. If this individual is susceptible when contacted then a local epidemic emanates from individual $\chi_{k+1}^{n}$. In particular, the local epidemic is constructed in an analogous fashion to $C_{k}^{n}$, translated so that the individual labelled 0 in $C_{k}^{n}$ corresponds to $\chi_{k}^{n}$. Note that the local spread away from $\chi_{i}^{n}$ may not be the same as that described by $C_{k}^{n}$, since in $E_{n}$ an individual that is susceptible in $C_{k}^{n}$ may have been infected via another infectious clump. Let $X_{n}(t)$ denote the total number of infectious clumps in $E_{n}$ at time $t$.

Let $T_{n}(\infty)$ denote the total number of infectious clumps ever present in the epidemic $E_{n}$, and let $T(\infty)$ denote the total progeny of the branching process.

Theorem 6.1. There is a probability space $(\Omega, \mathcal{F}, \mathcal{P})$ on which are defined a sequence of epidemic models indexed by $n$ (the population size) and the approximating branching process, with the following properties.

Denote by $A$ the set on which the branching process $X(\cdot)$ becomes extinct:

$$
A=\left\{\omega \in \Omega: \lim _{t \rightarrow \infty} X(t, \omega)=0\right\} .
$$

Then, as $n \rightarrow \infty$,

$$
\sup _{0 \leq t<\infty}\left|X_{n}(t)-X(t)\right| \rightarrow 0
$$

for $\mathcal{P}$-almost all $\omega \in A$. Furthermore, for any $0<K<\infty$, as $n \rightarrow \infty$,

$$
\sup _{0 \leq t \leq K}\left|X_{n}(t)-X(t)\right| \rightarrow 0
$$

for $\mathcal{P}$-almost all $\omega \in A^{\mathrm{c}}$.

Finally,

$$
T_{n}(\infty) \rightarrow T(\infty) \text { almost surely as } n \rightarrow \infty
$$


Proof. The proof follows along similar lines to [4, Theorem 2.5 and Corollary 2.6] and, hence, the details are omitted.

We briefly comment upon the differences with [4, Theorem 2.5]. The only technicality is that, in contrast to the SIR epidemic model, the local epidemics $\left\{C_{k}\right\}$ are not necessarily almost surely finite. However, arguments along the lines of Lemma 5.1 can be used to show that the rate of growth of the local epidemic $C_{k}$ is linear in $t$. Therefore, over any finite time interval, the arguments follow [4]. A weaker condition has been imposed upon the moments of $V$ than in [4, Theorem 2.5]. This is because (6.1) is stated for fixed $K$, with a stronger result in the vein of [4, Theorem 2.5] possible subject to higher finite moment constraints on $V$.

Theorem 6.1 gives us an invasion threshold theorem for the SIS great circle epidemic model. A global epidemic is said to occur if in the limit as $n \rightarrow \infty$ the epidemic generates infinitely many local infectious clumps. Let $R$ denote the total number of global infectious contacts emanating from a typical local epidemic, $C_{1}$. Note that $R \sim \operatorname{Po}\left(\lambda_{\mathrm{G}} \int_{0}^{\infty} I(t) \mathrm{d} t\right)$. By a time reversal argument (see [8] and [10]), $T \stackrel{\mathrm{D}}{=} \int_{0}^{\infty} I(t) \mathrm{d} t$, where $T$ denotes the susceptibility time; see Section 3. Thus, $R \sim \operatorname{Po}\left(\lambda_{\mathrm{G}} T\right)$ and $\mathrm{E}[R]=R_{*}$.

Corollary 6.1. Let $f(s)\left(=\mathrm{E}\left[s^{R}\right]\right)=\mathrm{E}\left[\exp \left((s-1) \lambda_{\mathrm{G}} T\right)\right]$ be the probability generating function of $R$. Then, as $n \rightarrow \infty$,

(a) a global epidemic occurs with nonzero probability if and only if $R_{*}>1$;

(b) the probability of a global epidemic is $p$, where $q=1-p$ is the smallest root of $\mathrm{E}\left[\exp \left((s-1) \lambda_{\mathrm{G}} T\right)\right]=s$ in $[0,1]$. Thus, $p=u^{*}$, where $u^{*}$ is the nonzero solution of (4.1).

An immediate consequence of Corollary 6.1(b) is that the probability of a global epidemic is identical to the proportion of the population infected in the endemic equilibrium. This holds generally for SIS models and is discussed further in Section 8.

Finally, unlike Sections 4 and 5, the above arguments can easily be adapted to the case where the infectious period is nonexponentially distributed; see, for example, [2, Section 2].

\section{Nearest neighbour}

The classical nearest neighbour contact process (with $\lambda_{\mathrm{G}}=0$ ) has received by far the most attention; see, for example, [6], [8], [9], and [10]. Assuming, without loss of generality, that $\gamma=1$, it is known that there exists $0<\lambda_{\mathrm{L}}^{C}<\infty$ such that, for all $\lambda_{\mathrm{L}} \leq \lambda_{\mathrm{L}}^{C}$, the contact process dies out almost surely, whilst, for $\lambda_{\mathrm{L}}>\lambda_{\mathrm{L}}^{C}$, an endemic equilibrium exists. The exact value of $\lambda_{\mathrm{L}}^{C}$ is unknown, although analytical arguments have shown that $1.539<\lambda_{\mathrm{L}}^{C} / 2<1.942$ and heuristic arguments give $\lambda_{\mathrm{L}}^{C} / 2 \approx 1.649$ [15]. (Note that the contact rate $\lambda$ which is usually quoted for contact processes (see, for example, [8], [10], and [15]) corresponds to $\lambda_{L} / 2$.)

Consider $\lambda_{\mathrm{G}}>0$, and let $T$ denote the susceptibility time of a typical individual; cf. Section 3 . Then, for $\lambda_{\mathrm{L}}<\lambda_{\mathrm{L}}^{C}, T$ is almost surely finite, whilst, for $\lambda_{\mathrm{L}}>\lambda_{\mathrm{L}}^{C}, \mathrm{P}(T=\infty)>0$. However, the distribution of $T$ is not known, and so, exact calculation of the proportion infected in endemic equilibrium $u^{*}$ is not possible. There are two fruitful approaches for evaluating $u^{*}$. The first approach is to derive analytical upper and lower bounds for $T$, and hence $u^{*}$. The second approach is heuristic and uses a self-consistent mean-field approximation. This approximation works very well when $\lambda_{G}$ is not too small $\left(\lambda_{G} \geq 0.25\right)$.

A lower bound for $T, T_{\mathrm{L}}$, is given by the SIR model, where individuals can be infected at most once. An upper bound, $T_{\mathrm{U}}$, assumes that all local infectious contacts result in infection. 
Thus,

$$
\begin{aligned}
& T_{\mathrm{L}} \stackrel{\mathrm{D}}{=} L+X\left(S_{L}^{1}+S_{L}^{2}\right), \\
& T_{\mathrm{U}} \stackrel{\mathrm{D}}{=} L+X\left(T_{\mathrm{U}}^{1}+T_{\mathrm{U}}^{2}\right),
\end{aligned}
$$

where all random variables in (7.1) and (7.2) are independent. Specifically, $L \sim \operatorname{Exp}\left(\lambda_{\mathrm{L}}+1\right)$, $X \sim \operatorname{Bernoulli}\left(\lambda_{\mathrm{L}} /\left(\lambda_{\mathrm{L}}+1\right)\right), T_{\mathrm{U}}^{1}$ and $T_{\mathrm{U}}^{2}$ are independent copies of $T_{\mathrm{U}}$, and $S_{L}^{1}$ and $S_{L}^{2}$ are independent copies of a random variable $S_{L}$, where

$$
S_{L} \stackrel{\mathrm{D}}{=} \tilde{\mathrm{L}}+\tilde{X}\left(\hat{\mathrm{L}}+S_{L}^{1}\right)
$$

with $\tilde{\mathrm{L}} \sim \operatorname{Exp}\left(1+\lambda_{\mathrm{L}} / 2\right), \hat{\mathrm{L}} \sim \operatorname{Exp}(1)$, and $\tilde{X} \sim \operatorname{Bernoulli}\left(\lambda_{\mathrm{L}} /\left(\lambda_{\mathrm{L}}+2\right)\right)$. Then, for $\alpha \geq 0$, straightforward calculations give

$$
\mathrm{E}\left[\exp \left(-\alpha T_{\mathrm{L}}\right)\right]=\frac{1}{\alpha+\lambda_{\mathrm{L}}+1}\left(1+\frac{\lambda_{\mathrm{L}}(\alpha+1)}{(\alpha+1)\left(\alpha+\lambda_{\mathrm{L}} / 2+1\right)-\lambda_{\mathrm{L}} / 2}\right)
$$

and

$$
\mathrm{E}\left[\exp \left(-\alpha T_{\mathrm{U}}\right)\right]=\frac{1}{2 \lambda_{\mathrm{L}}}\left(\alpha+\lambda_{\mathrm{L}}+1-\left(\left(\alpha+\lambda_{\mathrm{L}}+1\right)^{2}-8 \lambda_{\mathrm{L}}\right)^{1 / 2}\right) .
$$

Since $T_{\mathrm{L}} \leq_{\mathrm{st}} T \leq_{\mathrm{st}} T_{\mathrm{U}}$, we have, for all $\alpha \geq 0$,

$$
1-\mathrm{E}\left[\exp \left(-\lambda_{\mathrm{G}} \alpha T_{\mathrm{L}}\right)\right] \leq 1-\mathrm{E}\left[\exp \left(-\lambda_{\mathrm{G}} \alpha T\right)\right] \leq 1-\mathrm{E}\left[\exp \left(-\lambda_{\mathrm{G}} \alpha T_{\mathrm{U}}\right)\right] .
$$

Thus, $u_{\mathrm{L}} \leq u^{*} \leq u_{\mathrm{U}}$, where, for $Z=\mathrm{L}, \mathrm{U}, u_{Z}$ is the nonzero solution of

$$
u=1-\mathrm{E}\left[\exp \left(-\lambda_{\mathrm{G}} u T_{Z}\right)\right]
$$

if $\lambda_{\mathrm{G}} \mathrm{E}\left[T_{Z}\right]>1$ and $u_{Z}=0$ otherwise.

The self-consistent mean-field approach takes the following argument. Consider a random individual, $i$ say. In equilibrium a proportion $s$ of the population is infected and, thus, each individual is exposed to global infection at the points of a Poisson point process with rate $\lambda_{\mathrm{G}} s$. Therefore, if an individual has a constant number of infectious neighbours, $k$ say, the probability that individual $i$ is infectious at any given point in time is $\left(\lambda_{\mathrm{G}} s+k \lambda_{\mathrm{L}} / 2\right) /\left(1+\lambda_{\mathrm{G}} s+k \lambda_{\mathrm{L}} / 2\right)$ (the probability that the last event involving individual $i$ is an infectious contact). Moreover, for any individual, the probability that a given neighbour is infectious is $s$. Thus, the probability that individual $i$ is infectious is

$$
\sum_{k=0}^{2} \frac{\lambda_{\mathrm{G}} s+k \lambda_{\mathrm{L}} / 2}{1+\lambda_{\mathrm{G}} s+k \lambda_{\mathrm{L}} / 2}\left(\begin{array}{l}
2 \\
k
\end{array}\right) s^{k}(1-s)^{2-k} .
$$

However, the probability that individual $i$ is infectious is simply $s$. Therefore, the estimated proportion infectious in stationarity is $u_{H}$, where $u_{H}$ is the nonzero solution (should it exist) of

$$
s=\sum_{k=0}^{2} \frac{\lambda_{\mathrm{G}} s+k \lambda_{\mathrm{L}} / 2}{1+\lambda_{\mathrm{G}} s+k \lambda_{\mathrm{L}} / 2}\left(\begin{array}{l}
2 \\
k
\end{array}\right) s^{k}(1-s)^{2-k} .
$$

There are two assumptions implicit in the above arguments which are obviously incorrect. Firstly, the number of infectious neighbours is constant and, secondly, the infectious status of a neighbour of individual $i$ is independent of individual $i$ 's infectious state. However, if $\lambda_{\mathrm{G}} s$ 


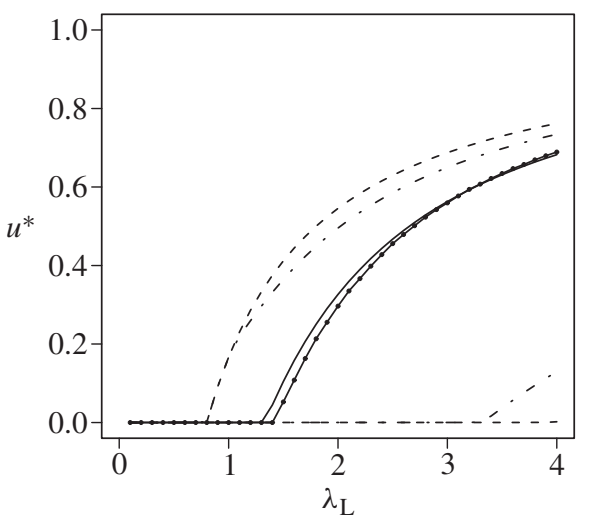

(a)

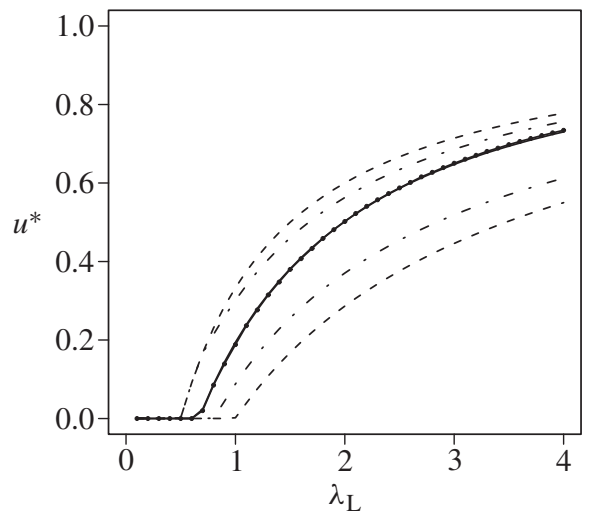

(b)

Figure 1: (a) $\lambda_{\mathrm{G}}=0.2$ and (b) $\lambda_{\mathrm{G}}=0.5$. The solid lines represent the simulation estimate of $u^{*}$ (with dots) and the self-consistent mean-field approximation (without dots). The dashed lines represent the upper and lower analytical bounds given by (7.3), and the dot-dash lines represent the tighter upper and lower analytical bounds.

is not small, an individual is only likely to escape infection if it has a short susceptibility time. Over short time intervals, it is reasonable to assume that the number of infectious neighbours is constant, and, thus, $u_{H}$ is potentially a good approximation for $u^{*}$, although the independence assumption is unjustified. Finally, for $\lambda_{G}=0,(7.5)$ has a nonzero solution if $\lambda_{L} / 2>\lambda_{L}^{C} / 2=1$, whereas the contact process is known to have $\lambda_{\mathrm{L}}^{C} / 2>1.539$.

We conclude with Figure 1, in which we compare the above estimates for $u^{*}$ with estimates of $u^{*}$ obtained from a simulation study on a population of size 10000 with the proportion infected averaged over 500 regularly observed time points. Also provided are tighter upper and lower analytical bounds for $C(\stackrel{\mathrm{D}}{=} T)$ based upon explicitly modelling the early stages of $C$ until either there are three infectious individuals or the clump has died out. The analytical upper and lower bounds are of limited use for $\lambda_{\mathrm{G}}$ small, although they do become far tighter as $\lambda_{\mathrm{G}}$ increases, where only individuals with short susceptibility times avoid infection. However, the self-consistent mean-field approach gives very good estimates for $u^{*}$ except in the case where $\lambda_{\mathrm{G}}$ and $u^{*}$ are small when it overestimates the proportion infected in stationarity.

\section{Summary}

The main results of the paper are given in Theorem 4.1 and Corollary 6.1 and can be summarised as follows. The probability, $p$, of a major epidemic outbreak (initiated from one initial infective) and the proportion, $u^{*}$, of the population infected in endemic equilibrium are the same. Moreover, $u^{*}=p$ is the nonzero solution (should one exist) of

$$
u=1-\mathrm{E}\left[\exp \left(-\lambda_{\mathrm{G}} u T\right)\right]
$$

where $T$ is the susceptibility time of a typical individual and a nonzero solution exists if and only if $R_{*}=\lambda_{\mathrm{G}} \mathrm{E}[T]>1$.

This result is by no means restricted to the great circle SIS epidemic model. For example, if $\lambda_{\mathrm{L}}=0$, we recover the standard SIS epidemic model with $T \sim \operatorname{Exp}(1)$. Therefore, a 
global epidemic is only possible if $\lambda_{\mathrm{G}}>1$ and the proportion of the population infectious in equilibrium is the nonzero solution of

$$
s=1-\frac{1}{\lambda_{\mathrm{G}} s+1} .
$$

That is, $u^{*}=\left(\lambda_{\mathrm{G}}-1\right) / \lambda_{\mathrm{G}}$, agreeing with [11].

More generally, the susceptibility time, $T$, of a typical individual can be defined for other two-level mixing SIS epidemic models. Of particular interest is the household model; see [2] and [14]. The proportion, $u^{*}$, of the population infectious in endemic equilibrium has been derived by different methods from those employed here; see [14, Section 4]. However, by using susceptibility times and arguing along the lines of Section 6, it can be shown that the probability of a major epidemic outbreak emanating from a single infective is also given by $u^{*}$.

Finally, the main drawback of the methodology is that the distribution of $T$ is difficult to derive. By restricting attention to the nearest neighbour model $\left(v_{1}=v_{-1}=\frac{1}{2}\right)$, stochastic upper and lower bounds for $T$ can be obtained. However, a self-consistent mean-field approach provided more useful approximations for $u^{*}$.

\section{Acknowledgement}

I would like to thank an anonymous referee for their careful reading of the paper and their comments which have greatly improved the presentation of the results.

\section{References}

[1] Andersson, H. (1999). Epidemic models and social networks. Math. Scientist 24, 128-147.

[2] BALL, F. (1999). Stochastic and deterministic models for SIS epidemics among a population partitioned into households. Math. Biosci. 156, 41-67.

[3] Ball, F. and Neal, P. (2002). A general model for stochastic SIR epidemics with two levels of mixing. Math. Biosci. 180, 73-102.

[4] Ball, F. and Neal, P. (2003). The great circle epidemic model. Stoch. Process. Appl. 107, $233-268$.

[5] Ball, F., Mollison, D. and Scalia-Tomba, G. (1997). Epidemics with two levels of mixing. Ann. Appl. Prob. 7, 46-89.

[6] Bezuidenhout, C. And Grimmett, G. (1990). The critical contact process dies out. Ann. Prob. 18, 1462-1482.

[7] Billingsley, P. (1968). Convergence of Probability Measures. John Wiley, New York.

[8] Griffeath, D. (1981). The basic contact processes. Stoch. Process. Appl. 11, 151-185.

[9] Harris, T. (1974). Contact interactions on a lattice. Ann. Prob. 2, 969-988.

[10] Harris, T. (1978). Additive set-valued Markov processes and graphical methods. Ann. Prob. 6, 355-378.

[11] Kryscio, R. And Lefèvre, C. (1989). On the extinction of the SIS stochastic logistic epidemic. J. Appl. Prob. 26, 685-694.

[12] Mollison, D. (1977). Spatial contact models for ecological and epidemic spread (with discussion). J. R. Statist. Soc. Ser. B 39, 283-326.

[13] Neal, P. (2006). Multitype randomised Reed-Frost epidemics and epidemics upon random graphs. Ann. Appl. Prob. 16, 1166-1189.

[14] Neal, P. (2006). Stochastic and deterministic analysis of SIS household epidemics. Adv. Appl. Prob. 38,943-968.

[15] Ricardo, J. and de Mendona, G. (1999). Precise critical exponents for the basic contact process. J. Phys. A Math. Gen. 32, 467-473. 